\title{
Gaussian Ensembles of Random Hermitian Matrices Intermediate Between Orthogonal and Unitary Ones
}

\author{
A. Pandey and M.L. Mehta
}

Service de Physique Théorique, Division de la Physique, CEN-Saclay, F-91191 Gif-sur-Yvette Cedex, France

\begin{abstract}
A Gaussian ensemble of Hermitian matrices depending on a parameter $\alpha$ is considered. When $\alpha=0$, the ensemble is Gaussian Orthogonal, and when $\alpha=1$, it is Gaussian Unitary. An analytic expression for the $n$-level correlation and cluster functions is given for any $n$ and $0 \leqq \alpha \leqq 1$. This ensemble is of relevance in the study of time reversal symmetry breaking of nuclear interactions.
\end{abstract}

\section{Introduction and Summary}

Wigner's Gaussian Orthogonal Ensemble (GOE) of asymptotically large real symmetric matrices has been the model for nuclear energy level fluctuations [1-4]. A large amount of data-mainly on slow-neutron resonances of medium and heavy nuclei, but also on some proton resonances of light nuclei-has been used to test the model. A very recent analysis indicates that the agreement between GOE and the data is remarkably good [5].

It can be shown [6] on general symmetry arguments that if the Hamiltonian governing the system is invariant under time reversal and space rotations, the appropriate ensemble is that of real symmetric matrices. On the other hand, if it contains a time reversal non-invariant part, then the matrices should have an imaginary part as well. One such ensemble, namely Gaussian Unitary Ensemble (GUE) of complex Hermitian matrices in which the real and imaginary parts are sampled independently but with the same weight, has also been studied analytically in great detail [1-4]. It gives very different fluctuation properties than the GOE and, in particular, has stronger level repulsion than observed in nuclear spectra, implying that the nuclear forces are mostly time reversal invariant. Following a suggestion of Wigner [2], the close agreement between GOE and the data may then be used to derive an upper bound on the time reversal non-invariant part of the nuclear interactions by studying ensembles of complex Hermitian matrices with a small imaginary part.

Ensembles of Hermitian matrices in which the dispersions of the real and imaginary parts are arbitrary and unequal have been the subject of several previous studies [7]. It is only recently, however, that approximate forms for the two-level 
correlation and cluster functions have been given [8]. It is made plausible that the transition from GOE to GUE in the (asymptotic) eigenvalue fluctuations is governed by a parameter $\lambda$ which is the dispersion of the imaginary part of the matrix elements expressed in local spacing units; see Ref. [9] for the relevance of this parameter in more general ensembles. In this paper we derive an exact closed expression for the $n$-level correlation and cluster functions for any $n$ for ensembles intermediate between GOE and GUE, and give, inter alia, a rigorous derivation of the transition parameter.

In what follows we consider an ensemble of Hermitian matrices depending on a parameter $\alpha$. The matrix elements are Gaussian random variables and the relative dispersion of their imaginary to real parts is $\alpha$. For $\alpha=0$, the ensemble is GOE, and for $\alpha=1$, it is GUE. We restrict ourselves to the case $0 \leqq \alpha \leqq 1$. This restriction enables us to write the ensemble as a sum of GOE and GUE, and consequently the joint probability density of the matrix elements as a convolution (Sect. 2). This plays a key role in the derivation of the joint probability density for the eigenvalues in Sect. 3.

The joint probability density for the eigenvalues follows easily in the two limiting cases from the invariance properties of the ensembles [10]; see Ref. [11] for the third limiting case $\alpha=\infty$. For arbitrary $\alpha$, however, one needs to integrate over the variables of the unitary transformations which diagonalize the matrices of the ensembles. For the restricted values of $\alpha$, the formula needed to do this is known [12]. Then, making use of the method of integration over alternate variables [13], we obtain the joint probability density as a pfaffian (Sect. 3).

The same method of integration can be used to derive the one-and-two-level functions [13]. We briefly discuss this line of inquiry in Appendix I. For more general results (Sect. 4), we rely on two theorems on quaternion determinants [14] which have already been used to derive all $n$-level functions for $\alpha=0,1$ [15].

In Sect. 5 we consider the limits when the dimension of the matrices is large. It is shown that the eigenvalue fluctuations (as described by the $n \geqq 2$-level functions) undergo a discontinuous transition at $\alpha=0$. For all non-zero finite $\alpha$ we obtain results characteristic of GUE. On the other hand, in the limit when $\alpha \rightarrow 0$ but the parameter $\lambda$ remains finite, the $n \geqq 2$-level functions undergo a smooth transition from $\operatorname{GOE}(\lambda=0)$ to $\operatorname{GUE}(\lambda=\infty)$. Except in the two limiting cases, the functions are non-stationary owing to the variation of $\lambda$ over the spectrum [8].

In Sect. 6 we study the (asymptotic) two-level functions in detail and derive expressions for the number variance $\Sigma^{2}$ as well as for the ensemble average of the least square statistic $\Delta_{3}$ which are useful in the analysis of data.

We do not consider here the data analysis for the breaking of time reversal symmetry, as it is already part of a separate study [8]. Some other quantities, such as the spacing distributions which follow from the cluster functions, will be discussed in a later paper.

\section{An Ensemble of Random Hermitian Matrices}

Consider the ensemble of Hermitian matrices

$$
H=A+B
$$


where $A$ is an $N \times N$ real symmetric matrix with the probability density

$$
\begin{gathered}
p_{1}(A)=2^{-N / 2}\left[2 \pi\left(1-\alpha^{2}\right) v^{2}\right]^{-N(N+1) / 4} \exp \left\{-\operatorname{Tr} A^{2} / 4\left(1-\alpha^{2}\right) v^{2}\right\}, \\
d A \equiv \prod_{i} d A_{i i} \prod_{j>k} d A_{j k},
\end{gathered}
$$

and $B$ an $N \times N$ Hermitian matrix with the probability density

$$
\begin{aligned}
p_{2}(B) & =2^{-N / 2}\left[2 \pi \alpha^{2} v^{2}\right]^{-N^{2} / 2} \exp \left\{-\operatorname{Tr} B^{2} / 4 \alpha^{2} v^{2}\right\}, \\
d B & =\prod_{i} d B_{i i} \prod_{j>k}\left(\operatorname{RedB} \mathrm{dB}_{j k}\right)\left(\operatorname{Im} d B_{j k}\right) .
\end{aligned}
$$

The joint probability density for the matrix elements of $H$ is then

and on the average

$$
\begin{aligned}
p(H) & =\int p_{1}(A) p_{2}(H-A) d A, \\
d H & =\prod_{i} d H_{i i} \prod_{j>k}\left(\operatorname{Re} d H_{j k}\right)\left(\operatorname{Im} d H_{j k}\right),
\end{aligned}
$$

$$
\frac{\|\operatorname{Im} H\|^{2}}{\|\operatorname{Re} H\|^{2}}=\left(\frac{N-1}{N+1}\right) \alpha^{2} \stackrel{\text { large } N}{\longrightarrow} \alpha^{2} .
$$

The ensemble is symmetric in $\alpha$. We consider therefore $\alpha \geqq 0$. Furthermore the definition (2.1) restricts it to $\alpha \leqq 1$. When $\alpha=0, B=0$ with probability one and then $H=A$ forms the GOE. Similarly $\alpha=1$ gives $H=B$ which is the GUE.

Here $v^{2}$ fixes the scale. We shall choose

$$
2 v^{2}\left(1+\alpha^{2}\right)=1,
$$

so that the results for GOE and GUE will be identical to those in [3].

We remark that the convolution integral in (2.6) can be evaluated easily and the resultant form, giving explicitly the joint probability density of the real and imaginary parts of $H$, is then valid for all $\alpha$. However, it is the integral form in (2.6), which will be used in the next section for the joint probability density of the eigenvalues.

\section{Joint Probability Density of the Eigenvalues}

Let $x_{1}, \ldots, x_{N}$ be the eigenvalues of $H$ :

$$
H=U^{\dagger} x U, x=\left[x_{i} \delta_{i j}\right], U^{\dagger} U=1,
$$

so that $[10]$

$$
d H=\left(\prod_{1}^{N} j !\right)^{-1} \pi^{N(N-1) / 2} \Delta^{2}(x) d x d U
$$

where

$$
\Delta(x)=\prod_{i>j}\left(x_{i}-x_{j}\right), d x \equiv d x_{1} \ldots d x_{N} .
$$


Similarly if $a_{1}, \ldots, a_{N}$ are the eigenvalues of $A$,

$$
A=\Omega^{T} a \Omega, a=\left[a_{i} \delta_{i j}\right], \Omega^{T} \Omega=1,
$$

then $[10]$

$$
d A=2^{-N}\left(\prod_{1}^{N} \Gamma\left(1+\frac{1}{2} j\right)\right)^{-1} \pi^{N(N+1) / 4}|\Delta(a)| d a d \Omega .
$$

To get the probability density $p(x)$ of the $x_{i}$, one has to further integrate over $U$ in Eq. (2.6). We have

$$
\begin{aligned}
p(x) & =\int p(H) \frac{d H}{d U} d U \\
& =\text { const } \Delta^{2}(x) \int \exp \left\{-\frac{\sum a_{i}^{2}}{4\left(1-\alpha^{2}\right) v^{2}}-\frac{\operatorname{Tr}\left(a-U^{\dagger} x U\right)^{2}}{4 \alpha^{2} v^{2}}\right\}|\Delta(a)| d a d U,
\end{aligned}
$$

where an intermediate step involves a trivial integration over $\Omega$. The constant in the last step is the product of the constants in $(2.2,2.4,3.2,3.5)$. The integral over $U$ can be performed by using the formula [12]

$\int d U \exp \left[-\frac{1}{2 t} \operatorname{Tr}\left(R-U^{\dagger} S U\right)^{2}\right]=t^{N(N-1) / 2} \prod_{0}^{N-1} j ! \frac{\operatorname{det}\left[\exp \left\{-\frac{1}{2 t}\left(r_{i}-s_{j}\right)^{2}\right\}\right]}{\Delta(r) \Delta(s)}$,

valid for Hermitian $R, S$ with eigenvalues $\left\{r_{i}\right\},\left\{s_{i}\right\}$. Note that the determinant has the same sign as $\Delta(r) \Delta(s)$. Then for the final integral over $a$, we have

$$
\begin{aligned}
& \int \exp \left(-\frac{\Sigma a_{i}^{2}}{4\left(1-\alpha^{2}\right) v^{2}}\right) \operatorname{det}\left[\exp \left\{-\frac{\left(a_{i}-x_{j}\right)^{2}}{4 \alpha^{2} v^{2}}\right\}\right] \operatorname{sign} \Delta(a) d a \\
& =N ! \exp \left(-\Sigma x_{i}^{2} / 4 v^{2}\right) \int d a \operatorname{det}\left[\exp \left\{-\frac{\left(a_{i}-\left(1-\alpha^{2}\right) x_{j}\right)^{2}}{4\left(1-\alpha^{2}\right) \alpha^{2} v^{2}}\right\}\right] \\
& \quad a_{1} \leqq a_{2} \leqq \cdots \leqq a_{N} \\
& =N ! \exp \left(-\Sigma x_{i}^{2} / 4 v^{2}\right) \operatorname{Pf}\left[\varepsilon\left(x_{i}, x_{j}\right)\right],
\end{aligned}
$$

where in the last step we have first integrated over alternate variables and then used the theory of Pfaffians [13]. To avoid minor complications, we have taken $N=2 \mathrm{~m}$, even. The antisymmetric function $\varepsilon(x, y)=-\varepsilon(y, x)$ is a double integral:

$$
\begin{aligned}
\varepsilon(x, y)= & \int_{-\infty}^{\infty} d z_{2} \int_{-x}^{z_{2}} d z_{1}\left\{\exp \left(-\frac{1}{4\left(1-\alpha^{2}\right) \alpha^{2} v^{2}}\left[\left(z_{1}-\left(1-\alpha^{2}\right) x\right)^{2}+\left(z_{2}-\left(1-\alpha^{2}\right) y\right)^{2}\right]\right)\right. \\
& \left.-\exp \left(-\frac{1}{4\left(1-\alpha^{2}\right) \alpha^{2} v^{2}}\left[\left(z_{1}-\left(1-\alpha^{2}\right) y\right)^{2}+\left(z_{2}-\left(1-\alpha^{2}\right) x\right)^{2}\right]\right)\right\} \\
= & 4 \pi\left(1-\alpha^{2}\right) \alpha^{2} v^{2} \operatorname{erf}\left\{\left(\frac{1-\alpha^{2}}{8 \alpha^{2} v^{2}}\right)^{1 / 2}(x-y)\right\}
\end{aligned}
$$


where

$$
\operatorname{erf}(x)=\frac{2}{\sqrt{ } \pi} \int_{0}^{x} \exp \left(-t^{2}\right) d t
$$

In the last step of (3.9) the integral over $z_{1}$ was performed after introducing the new variable $t=\left(z_{2}-z_{1}\right)$.

The Pfaffian in (3.8) is the square root of the determinant of the antisymmetric matrix $\varepsilon$. Collecting all the terms and using the normalization (2.9), we have finally, for even $N$,

$$
p(x)=C_{N} \exp \left(-\frac{1+\alpha^{2}}{2} \Sigma x_{i}^{2}\right)|\Delta(x)|\left\{\operatorname{det}\left[f\left(x_{i}-x_{j}\right)\right]\right\}^{1 / 2},
$$

with

$$
f(x)=\operatorname{erf}\left\{\left(\frac{1-\alpha^{4}}{4 \alpha^{2}}\right)^{1 / 2} x\right\}
$$

and

$$
C_{N}^{-1}=2^{3 N / 2}\left(1-\alpha^{2}\right)^{N(N-1) / 4}\left(1+\alpha^{2}\right)^{-N(N+1) / 4} \prod_{j=1}^{N} \Gamma\left(1+\frac{1}{2} j\right) .
$$

We remark parenthetically that the limit $\alpha=0$ is trivial since, then, $f(x)=\operatorname{sign} x$ and $\operatorname{det} f=1$. On the other hand for $\alpha=1$ one should expand $(\operatorname{det} f)^{1 / 2}$ in powers of $\left(\frac{1-\alpha^{4}}{4 \alpha^{2}}\right)^{1 / 2}$ to obtain

$$
\lim _{\alpha \rightarrow 1}\left(1-\alpha^{2}\right)^{-N(N-1) / 4}(\operatorname{det} f)^{1 / 2}=2^{N(N+3) / 4} \pi^{-N / 2} \prod_{j=1}^{N}\left(\frac{\Gamma\left(1+\frac{1}{2} j\right.}{j !}\right)|\Delta(x)| .
$$

In both cases the standard results $[10]$ are recovered.

\section{Correlation and Cluster Functions}

We shall use two theorems from the theory of quaternion matrices to derive the correlation and cluster functions. For a proof of the theorems, and details of the notions involved, one may consult either of the two references in [14]. The same theorems have been used to derive the functions for GOE and GUE [15]. An alternative derivation of the functions is outlined in Appendix $\mathrm{I}$.

Theorem 1. In an $N \times N$ self-dual quaternion matrix $\sigma$, replace its quaternion elements by their $2 \times 2$ matrix representatives as follows

$$
1 \rightarrow\left[\begin{array}{ll}
1 & 0 \\
0 & 1
\end{array}\right], e_{1} \rightarrow\left[\begin{array}{rr}
0 & -1 \\
1 & 0
\end{array}\right], e_{2} \rightarrow\left[\begin{array}{rr}
0 & -i \\
-i & 0
\end{array}\right], e_{3} \rightarrow\left[\begin{array}{rr}
i & 0 \\
0 & -i
\end{array}\right]
$$

This gives a matrix $C(\sigma)$ of twice the size with complex elements. Then

$$
\operatorname{det} C(\sigma)=\{Q \operatorname{det} \sigma\}^{2}
$$


where the quaternion-determinant $Q \operatorname{det} \sigma$ is defined as the scalar

$$
Q \operatorname{det} \sigma=\sum_{P}(-1)^{N-l} \prod_{1}^{l}\left(\sigma_{a b} \sigma_{b c} \cdots \sigma_{s a}\right) .
$$

Here $P$ is any permutation of the integers $(1,2, \ldots, N)$, consisting of l cycles of the form

$$
(a \rightarrow b \rightarrow c \rightarrow \cdots \rightarrow s \rightarrow a),
$$

and $(-1)^{N-l}$ is the parity of $P$.

Theorem 2. Let the quaternion elements $\sigma_{j k}$ of an $N \times N$ self-dual quaternion matrix $\sigma^{(N)}$ satisfy the following conditions:

$$
\sigma_{j k}=\Phi\left(x_{j}, x_{k}\right)=\bar{\sigma}_{k j},
$$

(where the bar denotes the operation of quaternion-adjoint), i.e. $\sigma_{j k}$ depends only on the variables $x_{j}$ and $x_{k}$,

$$
\begin{aligned}
\int \Phi(x, x) d \mu(x) & =c, \\
\int \Phi(x, y) \Phi(y, z) d \mu(y) & =\Phi(x, z)+\Phi_{1}(x, z), \\
\Phi_{1}(x, z) & =\tau \Phi(x, z)-\Phi(x, z) \tau,
\end{aligned}
$$

where $d \mu$ is a suitable measure, $c$ a constant scalar and $\tau$ a constant quaternion. Then

$$
\int Q \operatorname{det} \sigma^{(N)} d \mu\left(x_{N}\right)=(c-N+1) Q \operatorname{det} \sigma^{(N-1)},
$$

where $\sigma^{(N-1)}$ is the $(N-1) \times(N-1)$ matrix obtained from $\sigma^{(N)}$ by removing the row and column containing the variable $x_{N}$.

We exhibit now a self-dual quaternion matrix $\sigma^{(N)}\left(x_{1}, x_{2}, \ldots, x_{N}\right)$ and verify that its elements satisfy Eqs. (4.5-4.8). Moreover, we shall show that

$$
\operatorname{det} C\left(\sigma^{(N)}\right)=\{p(x)\}^{2},
$$

where $p(x)$ is given by (3.11). We can then immediately write down the $n$-level correlation function [16]

$$
R_{n}\left(x_{1}, x_{2}, \ldots, x_{n}\right)=\frac{N !}{(N-n) !} \int_{-\infty}^{\infty} \cdots \int p\left(x_{1}, \ldots, x_{N}\right) d x_{n+1} \cdots d x_{N}
$$

as a quaternion-determinant.

We write the quaternion elements of $\sigma$ by their $2 \times 2$ matrix representatives

$$
\sigma_{j k}=\Phi\left(x_{j}, x_{k}\right)=\left[\begin{array}{ll}
S_{N}\left(x_{j}, x_{k}\right) & D_{N}\left(x_{j}, x_{k}\right) \\
J_{N}\left(x_{j}, x_{k}\right) & S_{N}^{\dagger}\left(x_{j}, x_{k}\right)
\end{array}\right],
$$

where

$$
\begin{aligned}
S_{N}(x, y) & \equiv S_{N}^{\dagger}(y, x) \\
& =\sum_{j=0}^{m-1}\left\{\varphi_{2 j}(x) \varphi_{2 j}(y)-\psi_{2 j}(x) A_{2 j}(y)\right\}, \\
D_{N}(x, y) & =\sum_{j=0}^{m-1}\left\{\varphi_{2 j}(x) \psi_{2 j}(y)-\psi_{2 j}(x) \varphi_{2 j}(y)\right\}=-D_{N}(y, x),
\end{aligned}
$$




$$
\begin{aligned}
J_{N}(x, y) & =I_{N}(x, y)+g(x, y)=-J_{N}(y, x), \\
I_{N}(x, y) & =\sum_{j=0}^{m-1}\left\{\varphi_{2 j}(x) A_{2 j}(y)-A_{2 j}(x) \varphi_{2 j}(y)\right\}=-I_{N}(y, x), \\
g(x, y) & =\frac{1}{2}\left(\frac{1+\alpha^{2}}{1-\alpha^{2}}\right)^{1 / 2} e^{-(1 / 2) \alpha^{2}\left(x^{2}+y^{2}\right)} f(x-y)=-g(y, x),
\end{aligned}
$$

with

$$
\begin{aligned}
\varphi_{j}(x) & =\left(2^{j} j ! \sqrt{\pi}\right)^{-1 / 2} e^{-(1 / 2) x^{2}} H_{j}(x) \\
& =\left(2^{j} j ! \sqrt{\pi}\right)^{-1 / 2} e^{(1 / 2) x^{2}}\left(-\frac{d}{d x}\right)^{j} e^{-x^{2}}, \\
\psi_{j}(x) & =\left(\frac{1+\alpha^{2}}{1-\alpha^{2}}\right)^{j} e^{(1 / 2) \alpha^{2} x^{2}} \frac{d}{d x}\left\{e^{-(1 / 2) \alpha^{2} x^{2}} \varphi_{j}(x)\right\},
\end{aligned}
$$

and

$$
A_{j}(x)=\left(\frac{1-\alpha^{2}}{1+\alpha^{2}}\right)^{j} e^{-(1 / 2) \alpha^{2} x^{2}} \int_{-\infty}^{\infty} e^{(1 / 2) \alpha^{2} y^{2}} \in(x-y) \varphi_{j}(y) d y .
$$

Here $f(x)$ in (4.17) is as defined in (3.12), the $H_{j}(x)$ in (4.18) are the Hermite polynomials and $\in(x)$ in (4.20) is given by:

$$
\epsilon(x)=\frac{1}{2} \operatorname{sign} x \text {. }
$$

The $\varphi_{j}(x)$ are orthonormalized harmonic oscillator wave functions:

$$
\int_{-\infty}^{\infty} \varphi_{i}(x) \varphi_{j}(x) d x=\delta_{i j}
$$

By a partial integration we find that

$$
\int_{-\infty}^{\infty} A_{i}(x) \psi_{j}(x) d x=-\delta_{i j}
$$

Moreover, since the parity of $\varphi_{j}(x)$ is $(-1)^{j}$, while that of $\psi_{j}(x)$ or $A_{j}(x)$ is $(-1)^{j+1}$, we have

$$
\int_{-\infty}^{\infty} \varphi_{i}(x) \psi_{j}(x) d x=\int_{-\infty}^{\infty} \varphi_{i}(x) A_{j}(x) d x=0, \quad i+j=\text { even. }
$$

We now define, for any two functions $f_{1}(x, y)$ and $f_{2}(x, y)$, the composition

$$
f_{1} * f_{2} \equiv\left(f_{1} * f_{2}\right)(x, z) \equiv \int_{-\infty}^{\infty} f_{1}(x, y) f_{2}(y, z) d y
$$

Then using (4.22-4.24) we find that

$$
\begin{aligned}
& S_{N} * S_{N}=S_{N}, \\
& S_{N} * D_{N}=D_{N} * S_{N}^{\dagger}=D_{N},
\end{aligned}
$$




$$
I_{N} * S_{N}=S_{N}^{\dagger} * I_{N}=I_{N}
$$

and

$$
I_{N} * D_{N}=S_{N}^{\dagger}, \quad D_{N} * I_{N}=S_{N} .
$$

To evaluate $g * S_{N}$ and $g * D_{N}$ we need the integrals (see Appendix II):

$$
\int_{-\infty}^{\infty} g(x, y) \psi_{j}(y) d y=\varphi_{j}(x)
$$

and

$$
\int_{-\infty}^{\infty} g(x, y) \varphi_{j}(y) d y=A_{j}(x)
$$

Using these equations we get:

$$
g * S_{N}=S_{N}^{\dagger} * g=-I_{N}
$$

and

$$
g * D_{N}=-S_{N}^{\dagger}, \quad D_{N} * g=-S_{N},
$$

so that

$$
\begin{aligned}
& J_{N} * S_{N}=S_{N}^{\dagger} * J_{N}=0, \\
& J_{N} * D_{N}=D_{N} * J_{N}=0
\end{aligned}
$$

Thus

$$
\begin{aligned}
\Phi * \Phi & =\left[\begin{array}{ll}
S_{N} * S_{N}+D_{N} * J_{N} & S_{N} * D_{N}+D_{N} * S_{N}^{\dagger} \\
J_{N} * S_{N}+S_{N}^{\dagger} * J_{N} & J_{N} * D_{N}+S_{N}^{\dagger} * S_{N}^{\dagger}
\end{array}\right] \\
& =\left[\begin{array}{cc}
S_{N} & 2 D_{N} \\
0 & S_{N}^{\dagger}
\end{array}\right]=\Phi+\Phi_{1},
\end{aligned}
$$

where

$$
\Phi_{1}=\left[\begin{array}{cc}
0 & D_{N} \\
-J_{N} & 0
\end{array}\right]=\tau \Phi-\Phi \tau,
$$

with

$$
\tau=\frac{1}{2}\left[\begin{array}{rr}
1 & 0 \\
0 & -1
\end{array}\right]
$$

We also see from $(4.22,4.23)$ that

$$
\int_{-\infty}^{\infty} \Phi(x, x) d x=\int_{-\infty}^{\infty} S_{N}(x, x) d x=2 m=N .
$$

Thus Eqs. (4.5-4.8) are satisfied with $c=N$ and $\tau$ given by (4.38). 
To verify (4.10) we observe that the $2 N \times 2 N$ matrix

$$
\begin{aligned}
G & =\left[\begin{array}{ll}
S_{N}\left(x_{j}, x_{k}\right) & D_{N}\left(x_{j}, x_{k}\right) \\
I_{N}\left(x_{j}, x_{k}\right) & S_{N}^{\dagger}\left(x_{j}, x_{k}\right)
\end{array}\right] \\
& =\left[\begin{array}{ccc}
\varphi_{2 i}\left(x_{j}\right) & -\psi_{2 i}\left(x_{j}\right) & 0 \\
-A_{2 i}\left(x_{j}\right) & \varphi_{2 i}\left(x_{j}\right) & 0
\end{array}\right]\left[\begin{array}{cc}
\varphi_{2 i}\left(x_{k}\right) & \psi_{2 i}\left(x_{k}\right) \\
A_{2 i}\left(x_{k}\right) & \varphi_{2 i}\left(x_{k}\right) \\
0 & 0
\end{array}\right]
\end{aligned}
$$

has rank $N$. In other words, the last $N$ rows

$$
\left[I_{N}\left(x_{j}, x_{k}\right) S_{N}^{\dagger}\left(x_{j}, x_{k}\right)\right]
$$

are linear combinations of the first $N$ rows

$$
\left[S_{N}\left(x_{j}, x_{k}\right) D_{N}\left(x_{j}, x_{k}\right)\right] \text {. }
$$

The determinant of $C\left(\sigma^{(N)}\right)$,

$$
C\left(\sigma^{(N)}\right)=\left[\begin{array}{ll}
S_{N}\left(x_{j}, x_{k}\right) & D_{N}\left(x_{j}, x_{k}\right) \\
J_{N}\left(x_{j}, x_{k}\right) & S_{N}^{\dagger}\left(x_{j}, x_{k}\right)
\end{array}\right],
$$

is therefore not changed if we subtract from its last $N$ rows the last $N$ rows of $G$. Thus

$$
\begin{aligned}
\operatorname{det} C\left(\sigma^{(N)}\right) & =\operatorname{det}\left[\begin{array}{ll}
S_{N}\left(x_{j}, x_{k}\right) & D_{N}\left(x_{j}, x_{k}\right) \\
g\left(x_{j}, x_{k}\right) & 0
\end{array}\right] \\
& =(-1)^{N} \operatorname{det}\left[g\left(x_{j}, x_{k}\right)\right] \operatorname{det}\left[D_{N}\left(x_{j}, x_{k}\right)\right] .
\end{aligned}
$$

Moreover, from (4.17) we have

$$
\operatorname{det}\left[g\left(x_{j}, x_{k}\right)\right]=2^{-N}\left(\frac{1+\alpha^{2}}{1-\alpha^{2}}\right)^{N / 2} e^{-\alpha^{2} \Sigma x_{\imath}^{2}} \operatorname{det}\left[f\left(x_{j}-x_{k}\right)\right],
$$

and, since

$$
\left[D_{N}\left(x_{j}, x_{k}\right)\right]=\left[\varphi_{2 i}\left(x_{j}\right) \psi_{2 i}\left(x_{j}\right)\right]\left[\begin{array}{c}
\psi_{2 i}\left(x_{k}\right) \\
-\varphi_{2 i}\left(x_{k}\right)
\end{array}\right],
$$

we also have from $(4.18,4.19)$

$$
\begin{aligned}
\operatorname{det}\left[D_{N}\left(x_{j}, x_{k}\right)\right]= & (-1)^{N}\left\{\operatorname{det}\left[\varphi_{2 i}\left(x_{j}\right) \psi_{2 i}\left(x_{j}\right)\right]\right\}^{2} \\
= & (-1)^{N}\left(\prod_{k=0}^{m-1}\left(\frac{2 k+1}{2}\right)\right)\left(\frac{1+\alpha^{2}}{1-\alpha^{2}}\right)^{N(N-2) / 2}\left(1+\alpha^{2}\right)^{N}\left\{\operatorname{det}\left[\varphi_{i}\left(x_{j}\right)\right]\right\}^{2} \\
= & (-1)^{N} 2^{-2 N}\left(\prod_{j=1}^{N} \Gamma\left(1+\frac{1}{2} j\right)\right)^{-2} \\
& \times\left(1-\alpha^{2}\right)^{-N(N-2) / 2}\left(1+\alpha^{2}\right)^{N^{2} / 2} e^{-\Sigma x_{i}^{2}} \Delta^{2}(x),
\end{aligned}
$$

where in the last two steps we have made repeated use of the fact that a determinant is not changed if we add to a column a linear combination of the other columns. 
Thus in the second step we have used the relation

$$
\sqrt{2} \psi_{j}(x)=\left(\frac{1+\alpha^{2}}{1-\alpha^{2}}\right)^{j}\left\{\sqrt{j}\left(1-\alpha^{2}\right) \varphi_{j-1}(x)-\sqrt{j+1}\left(1+\alpha^{2}\right) \varphi_{j+1}(x)\right\}
$$

to replace the column $\psi_{2 i}$ by $\varphi_{2 i+1}$. (Note that in the first step the index $i$ runs from zero to $m-1$ whereas in the second from zero to $N-1$; in both steps $j$ is from 1 to $N$.) The last form follows by reducing $\operatorname{det}\left[H_{i}\left(x_{j}\right)\right]$ to $\operatorname{det}\left[x_{j}^{i}\right]$ which is the Vandermonde determinant $\Delta(x)$. Equations $(4.44,4.45,4.47)$ now give (4.10).

All the conditions of the theorem being satisfied, we apply it several times to obtain from (4.9-4.11)

$$
R_{n}\left(x_{1}, x_{2}, \ldots, x_{n}\right)=Q \operatorname{det} \sigma^{(n)}=\left\{\operatorname{det} C\left(\sigma^{(n)}\right)\right\}^{1 / 2},
$$

where $\sigma^{(n)}$ is the $n \times n$ quaternion matrix

$$
\sigma^{(n)} \equiv \sigma^{(n)}\left(x_{1}, x_{2}, \ldots, x_{n}\right)=\left[\Phi\left(x_{j}, x_{k}\right)\right]_{j, k=1, \ldots, n},
$$

and $C\left(\sigma^{(n)}\right)$ is the $2 n \times 2 n$ matrix

$$
C\left(\sigma^{(n)}\right)=\left[\begin{array}{ll}
S_{N}\left(x_{j}, x_{k}\right) & D_{N}\left(x_{j}, x_{k}\right) \\
J_{N}\left(x_{j}, x_{k}\right) & S_{N}^{\dagger}\left(x_{j}, x_{k}\right)
\end{array}\right]_{j, k=1,2, \ldots, n}
$$

Thus the level density is

$$
\begin{aligned}
R_{1}(x) & =S_{N}(x, x) \\
& =\sum_{j=0}^{N-1} \varphi_{j}^{2}(x)+\left(1-\alpha^{2}\right) \sqrt{N / 2} \varphi_{N-1}(x) e^{-(1 / 2) \alpha^{2} x^{2}} \int_{-\infty}^{\infty} \in(x-z) e^{(1 / 2) \alpha^{2} z^{2}} \varphi_{N}(z) d z
\end{aligned}
$$

where the last form follows from the simplified expression for $S_{N}$ given in (4.63) below. The two-level correlation function is

$$
R_{2}(x, y)=R_{1}(x) R_{1}(y)-T_{2}(x, y),
$$

where the two-level cluster function $T_{2}$ is given by

$$
T_{2}(x, y)=S_{N}(x, y) S_{N}(y, x)-D_{N}(x, y) J_{N}(x, y) .
$$

As in $[14,15]$ the $n$-level cluster function is given by

$$
T_{n}\left(x_{1}, x_{2}, \ldots, x_{n}\right)=\sum_{P} \Phi\left(x_{1}, x_{2}\right) \Phi\left(x_{2}, x_{3}\right) \ldots \Phi\left(x_{n}, x_{1}\right),
$$

where the sum is taken over all the $(n-1)$ ! distinct cyclic permutations of the indices $(1,2, \ldots, n)$. The functions satisfy the integral relations, namely

$$
\int_{-\infty}^{\infty} R_{n}\left(x_{1}, x_{2}, \ldots, x_{n}\right) d x_{n}=(N-n+1) R_{n-1}\left(x_{1}, x_{2}, \ldots, x_{n-1}\right),
$$

and

$$
\int_{-\infty}^{\infty} T_{n}\left(x_{1}, x_{2}, \ldots, x_{n}\right) d x_{n}=(n-1) T_{n-1}\left(x_{1}, x_{2}, \ldots, x_{n-1}\right)
$$


with

$$
\int_{-\infty}^{\infty} R_{1}(x) d x=\int_{-\infty}^{\infty} T_{1}(x) d x=N
$$

as they should [16].

One can use (4.48) and

$$
\sqrt{2} \varphi_{j}(x)=\left(\frac{1+\alpha^{2}}{1-\alpha^{2}}\right)^{j}\left\{\sqrt{j}\left(1-\alpha^{2}\right) A_{j-1}(x)-\sqrt{j+1}\left(1+\alpha^{2}\right) A_{j+1}(x)\right\}
$$

to simplify the expressions for $S_{N}$ and $J_{N}$. We have

$$
\begin{aligned}
\psi_{2 j}(x) A_{2 j}(y)= & -\varphi_{2 j+1}(x) \varphi_{2 j+1}(y)+\left(1-\alpha^{2}\right)\left\{\left(\frac{1+\alpha^{2}}{1-\alpha^{2}}\right)^{2 j} \sqrt{j} \varphi_{2 j-1}(x) A_{2 j}(y)\right. \\
& \left.-\left(\frac{1+\alpha^{2}}{1-\alpha^{2}}\right)^{2 j+2} \sqrt{j+1} \varphi_{2 j+1}(x) A_{2 j+2}(y)\right\}, \\
\varphi_{2 j}(x) A_{2 j}(y)= & -A_{2 j+1}(x) \varphi_{2 j+1}(y)+\left(1-\alpha^{2}\right)\left\{\left(\frac{1+\alpha^{2}}{1-\alpha^{2}}\right)^{2 j} \sqrt{j} A_{2 j-1}(x) A_{2 j}(y)\right. \\
& \left.-\left(\frac{1+\alpha^{2}}{1-\alpha^{2}}\right)^{2 j+2} \sqrt{j+1} A_{2 j+1}(x) A_{2 j+2}(y)\right\},
\end{aligned}
$$

and

$$
\begin{aligned}
\psi_{2 j}(x) \varphi_{2 j}(y)= & -\varphi_{2 j+1}(x) \psi_{2 j+1}(y)+\left(1-\alpha^{2}\right)\left\{\left(\frac{1+\alpha^{2}}{1-\alpha^{2}}\right)^{2 j} \sqrt{j} \varphi_{2 j-1}(x) \varphi_{2 j}(y)\right. \\
& \left.-\left(\frac{1+\alpha^{2}}{1-\alpha^{2}}\right)^{2 j+2} \sqrt{j+1} \varphi_{2 j+1}(x) \varphi_{2 j+2}(y)\right\}
\end{aligned}
$$

Then from $(4.13,4.60)$

$$
\begin{aligned}
S_{N}(x, y)= & \sum_{j=0}^{2 m-1} \varphi_{j}(x) \varphi_{j}(y)+\left(1-\alpha^{2}\right) \sqrt{m} \varphi_{N-1}(x) e^{-(1 / 2) \alpha^{2} x^{2}} \\
& \times \int_{-\infty}^{\infty} \in(y-z) e^{(1 / 2) \alpha^{2} z^{2}} \varphi_{N}(z) d z .
\end{aligned}
$$

Also, from $(4.31,4.61)$ we have

$$
\begin{aligned}
g(x, y) & =\sum_{j=0}^{\infty} A_{j}(x) \varphi_{j}(y)=\frac{1}{2} \sum_{j=0}^{\infty}\left\{A_{j}(x) \varphi_{j}(y)-\varphi_{j}(x) A_{j}(y)\right\} \\
& =\sum_{j=0}^{\infty}\left\{A_{2 j}(x) \varphi_{2 j}(y)-\varphi_{2 j}(x) A_{2 j}(y)\right\} \\
& =\sum_{j=0}^{\infty}\left\{A_{2 j+1}(x) \varphi_{2 j+1}(y)-\varphi_{2 j+1}(x) A_{2 j+1}(y)\right\}
\end{aligned}
$$


which along with $(4.15,4.16)$ gives

$$
J_{N}(x, y)=\sum_{j=m}^{\infty}\left\{A_{2 j}(x) \varphi_{2 j}(y)-\varphi_{2 j}(y) A_{2 j}(x)\right\} .
$$

The result (4.55) is already in the form given for $\alpha=0,1$ in [15]. For $\alpha=0$, $g(x, y)=\epsilon(x-y)$, and $D_{N}$ and $I_{N}$ can be written, with the help of $(4.61,4.62)$, as a differential and an integral, respectively, of $S_{N}$ in (4.63). On the other hand for $\alpha=1$, while $D_{N}$ diverges, its product with $J_{N},(4.65)$, is zero so that only $S_{N}$ terms survive in (4.55).

\section{Limits when the Matrices are Large}

The large- $N$ limits of the $n$-level functions are known $[15,17]$ for $\alpha=0,1$. We follow here the method of [17] where the limits for $n(>1)$-level functions are derived for every region of the spectrum.

We have, from (4.63), for large $N$

$$
\begin{aligned}
S_{N}(x, y) & \approx \sum_{j=0}^{N-1} \varphi_{j}(x) \varphi_{j}(y) \\
& =\left(\frac{1}{2} N\right)^{1 / 2}\left\{\frac{\varphi_{N}(x) \varphi_{N-1}(y)-\varphi_{N}(y) \varphi_{N-1}(x)}{x-y}\right\} \\
& \approx \frac{(2 N)^{1 / 2} \sin \theta}{\pi}\left\{\frac{\sin \left(2 N \delta \theta \sin ^{2} \theta\right)}{2 N \delta \theta \sin ^{2} \theta}\right\} .
\end{aligned}
$$

Here the second form is obtained by the Christoffel-Darboux formula and the last form, valid for small $\delta \theta$, by using the asymptotic formula [18] for $\varphi_{N}$,

$$
\varphi_{N}(x)=\left(\frac{2}{N \pi^{2} \sin ^{2} \theta}\right)^{1 / 4}\left[\sin \left\{\left(\frac{2 N+1}{4}\right)(2 \theta-\sin 2 \theta)-\left(\frac{4 N-1}{4}\right) \pi\right\}+O\left(N^{-1}\right)\right]
$$

where

$$
\theta \equiv \theta(x)=\cos ^{-1}\left(-\frac{x}{\sqrt{2 N+1}}\right), \quad 0<\theta<\pi
$$

and

$$
\delta \theta=\theta(x)-\theta(y) \approx(x-y) /(2 N)^{1 / 2} \sin \theta .
$$

Thus for the asymptotic level density, we obtain from $(4.52,5.1)$

$$
R_{1}(x) \approx \frac{(2 N)^{1 / 2} \sin \theta}{\pi} \approx \pi^{-1}\left(2 N-x^{2}\right)^{1 / 2},
$$

the famous semicircle of Wigner. Also, as $N \rightarrow \infty,(x-y) \rightarrow 0$ while

$$
r=(x-y) R_{1}(x) \approx \frac{2 N \delta \theta \sin ^{2} \theta}{\pi}
$$


remains finite, we have from (5.1)

$$
S_{N}(x, y) \rightarrow R_{1}(x) \frac{\sin \pi r}{\pi r} .
$$

For non-zero finite $\alpha$, we see from Eqs. $(4.14,4.19,4.20,4.65)$ that

$$
D_{N}(x, y) \rightarrow \infty, \quad J_{N}(x, y) \rightarrow 0,
$$

and

$$
D_{N}(x, y) J_{N}(x, y) \rightarrow 0,
$$

implying that in the asymptotic- $N$ limit the $n$-level correlation and cluster functions for $n>1$ are discontinuous functions of $\alpha$. For $\alpha=0$ we have the GOE results and for $\alpha>0$ the GUE results. However, $D_{N}$ and $J_{N}$ have finite limits when $\alpha \rightarrow 0$ and

$$
\lambda \equiv \lambda(x)=\frac{\left(\operatorname{Im} H_{i j}\right)^{\mathrm{R} M \mathrm{~S}}}{\text { Local Average Spacing at } x} \approx \frac{\alpha}{\sqrt{2}} R_{1}(x)
$$

is finite [8]. These limits can be obtained by taking the Fourier transform of Eqs. (4.32, 4.33), using (5.7) for $S_{N}$. Using the notation

$$
\mathscr{F}(h(x)) \equiv \int_{-\infty}^{\infty} e^{2 \pi i k x} h(x) d x
$$

for the Fourier transform of a function $h(x)$, we have [19] for $b>0$

$$
\mathscr{F}(\operatorname{erf}(b x))=2 i \int_{0}^{\infty} \sin (2 \pi k x) \operatorname{erf}(b x) d x=\frac{i}{\pi k} \exp \left(-\pi^{2} k^{2} / b^{2}\right),
$$

and

$$
\mathscr{F}\left(\frac{\sin b x}{x}\right)=2 \int_{0}^{\infty} \cos (2 \pi k x) \frac{\sin b x}{x} d x=\left\{\begin{array}{cl}
\pi, & \text { if } 2 \pi|k|<b \\
\pi / 2 & , \text { if } 2 \pi|k|=b \\
0, & \text { if } 2 \pi|k|>b
\end{array}\right.
$$

which, along with (4.32), give for the Fourier transform of $I_{N}$.

$$
\mathscr{F}\left(I_{N}\right) \approx \frac{i}{2 \pi k} \exp \left(-8 \pi^{2} \lambda^{2} k^{2}\right)\left\{\begin{array}{cl}
1 & , \text { if }|k|<1 / 2 \\
1 / 2, & \text { if }|k|=1 / 2 \\
0, & \text { if }|k|>1 / 2
\end{array}\right.
$$

An inverse Fourier transform then gives

$$
I_{N}(x, y) \approx-\frac{1}{\pi} \int_{0}^{r} d t \int_{0}^{\pi} d k \cos k t e^{-2 \lambda^{2} k^{2}}=-\frac{1}{\pi} \int_{0}^{\pi} d k \frac{\sin k r}{k} e^{-2 \lambda^{2} k^{2}},
$$

so that

$$
J_{N}(x, y) \approx J(r ; \lambda)=-\frac{1}{\pi} \int_{0}^{r} d t \int_{\pi}^{\infty} d k \cos k t e^{-2 \lambda^{2} k^{2}}=-\frac{1}{\pi} \int_{\pi}^{\infty} d k \frac{\sin k r}{k} e^{-2 \lambda^{2} k^{2}} .
$$


Similarly from (4.33) we have

$$
\begin{aligned}
D_{N}(x, y) & \approx\left(R_{1}(x)\right)^{2} D(r ; \lambda), \\
D(r ; \lambda) & =\frac{d}{d r}\left\{\frac{1}{\pi} \int_{0}^{\pi} d k \cos k r e^{2 \lambda^{2} k^{2}}\right\}=-\frac{1}{\pi} \int_{0}^{\pi} d k k \sin k r e^{2 \lambda^{2} k^{2}} .
\end{aligned}
$$

Using the new variables

$$
r_{i}=\int_{-\infty}^{x_{1}} R_{1}(x) d x
$$

we get, from $(4.49-4.51,4.55,5.7,5.16-5.18)$, for the $n$-level correlation function

$$
\begin{aligned}
\mathscr{R}_{n}\left(r_{1}, r_{2}, \ldots r_{n} ; \lambda\right) & \equiv \lim _{N \rightarrow \infty}\left\{R_{1}\left(x_{1}\right) R_{1}\left(x_{2}\right) \cdots R_{1}\left(x_{n}\right)\right\}^{-1} R_{n}\left(x_{1}, x_{2}, \ldots, x_{n}\right) \\
& =Q \operatorname{det}\left[\Phi\left(r_{i}-r_{j} ; \lambda\right)\right]_{i, j=1,2, \ldots, n},
\end{aligned}
$$

and for the $n$-level cluster function

$$
\begin{aligned}
Y_{n}\left(r_{1}, r_{2}, \ldots, r_{n} ; \lambda\right) & \equiv \lim _{N \rightarrow \infty}\left\{R_{1}\left(x_{1}\right) R_{1}\left(x_{2}\right) \cdots R_{1}\left(x_{n}\right)\right\}^{-1} T_{n}\left(x_{1}, x_{2}, \ldots, x_{n}\right) \\
& =\sum_{P} \Phi\left(r_{1}-r_{2} ; \lambda\right) \Phi\left(r_{2}-r_{3} ; \lambda\right) \cdots \Phi\left(r_{n}-r_{1} ; \lambda\right)
\end{aligned}
$$

where

$$
\Phi(r ; \lambda)=\left[\begin{array}{cc}
\frac{\sin \pi r}{\pi r} & D(r ; \lambda) \\
J(r ; \lambda) & \frac{\sin \pi r}{\pi r}
\end{array}\right],
$$

with $J(r)$ and $D(r)$ given above, Eqs. $(5.16,5.18)$.

The transition parameter $\lambda$, Eq. (5.1), varies over the spectrum because of its dependence on $x$. As a consequence, the fluctuation properties, described by the set of $\mathscr{R}_{n}$ or $Y_{n}$ functions for $n>1$, are non-stationary, the exceptions [17] being the two limiting cases. In particular, the $\operatorname{GOE}(\lambda=0) \rightarrow \operatorname{GUE}(\lambda=\infty)$ transition then is seen to be faster in the middle of the semicircle than at the ends [8].

\section{Two-Point Fluctuation Measures}

The two-level cluster function, namely

$$
Y_{2}(r ; \lambda)=\left(\frac{\sin \pi r}{\pi r}\right)^{2}-J(r ; \lambda) D(r ; \lambda)=Y_{2}(r ; \infty)-J(r ; \lambda) D(r ; \lambda),
$$

is of particular interest in the analysis of data. Its asymptotic expansion can be derived by partial integration in $(5.16,5.18)$. We have for $|r| \gtrsim 1$

$$
Y_{2}(r ; \lambda)-Y_{2}(r ; \infty)=\frac{r^{2} \cos ^{2} \pi r-16 \pi^{2} \lambda^{4} \sin ^{2} \pi r}{\pi^{2}\left(r^{2}+16 \pi^{2} \lambda^{4}\right)^{2}}+\ldots
$$


valid for any value of $\lambda$ whether large or small compared to $r$ (see appendix III). For the Fourier transform,

$$
b(k ; \lambda)=\int_{-\infty}^{\infty} e^{2 \pi i k r} Y_{2}(r ; \lambda) d r
$$

we have $[20]$

$$
b(k ; \infty)=\int_{-\infty}^{\infty}\left(\frac{\sin \pi r}{\pi r}\right)^{2} e^{2 \pi i k r} d r=\left\{\begin{array}{cc}
1-|k|, & |k| \leqq 1, \\
0, & |k| \geqq 1,
\end{array}\right.
$$

and

$$
\begin{aligned}
& b(k ; \lambda)-b(k ; \infty)=-\int_{-\infty}^{\infty} J(r ; \lambda) D(r ; \lambda) \exp (2 \pi i k r) d r \\
= & -\int_{-\infty}^{\infty} d r \int_{1}^{\infty} d k_{1} \int_{0}^{1} d k_{2} \frac{k_{2}}{k_{1}} \sin \left(\pi k_{1} r\right) \sin \left(\pi k_{2} r\right) \exp (2 \pi i k r) \\
& \times \exp \left[-2 \pi^{2} \lambda^{2}\left(k_{1}^{2}-k_{2}^{2}\right)\right] \\
= & \frac{1}{4} \int_{0}^{\infty} d k_{1} \int_{-1}^{1} d k_{2} \frac{k_{2}}{k_{1}} \delta\left(|k|-\frac{k_{1}+k_{2}}{2}\right) \exp \left[-2 \pi^{2} \lambda^{2}\left(k_{1}^{2}-k_{2}^{2}\right)\right] .
\end{aligned}
$$

Integrating over $k_{1}$ we get

$$
\begin{aligned}
& b(k ; \lambda)-b(k ; \infty) \\
& =\left\{\begin{array}{l}
-\frac{1}{2}|k| \exp \left(-8 \pi^{2} \lambda^{2}|k|\right) \int_{-1}^{1} \frac{t-1+1 /|k|}{t+1+1 / k} \exp \left(-8 \pi^{2} \lambda^{2} k^{2} t\right),|k| \leqq 1, \\
-\frac{1}{2} \exp \left(-8 \pi^{2} \lambda^{2} k^{2}\right) \int_{-1}^{1} d t \frac{t}{t+2|k|} \exp \left(-8 \pi^{2} \lambda^{2}|k| t\right), \quad|k| \geqq 1,
\end{array}\right.
\end{aligned}
$$

while integrating over $k_{2}$ we get a different form,

where

$$
b(k ; \lambda)-b(k ; \infty)=-k e^{-8 \pi^{2} \lambda^{2}|k|}\{1+h(k ; \lambda)\},
$$

$$
1+h(k ; \lambda)= \begin{cases}\frac{\operatorname{sh}\left(8 \pi^{2} \lambda^{2} k^{2}\right)}{8 \pi^{2} \lambda^{2} k^{2}}+E(1,2|k|+1), & |k| \leqq 1, \\ \frac{\operatorname{sh}\left(8 \pi^{2} \lambda^{2}|k|\right)}{8 \pi^{2} \lambda^{2} k^{2}} e^{8 \pi^{2} \lambda^{2}\left(|k|-k^{2}\right)}+E(2|k|-1,2|k|+1), & |k| \geqq 1,\end{cases}
$$

and

$$
E(x, y)=\exp \left(8 \pi^{2} \lambda^{2}\left(k^{2}+|k|\right)\right) \int_{y}^{x} \exp \left(-8 \pi^{2} \lambda^{2}|k| t\right) \frac{d t}{t} .
$$

which can of course be expressed in terms of the standard $E_{1}$ functions.

A quantity often used to measure the rigidity of the spectrum is the number variance $\Sigma^{2}(\bar{n})$, namely the variance of the number of levels in a given interval containing on the average $\bar{n}$ levels $[21,22]$. We have in the present case

$$
\Sigma^{2}(\bar{n} ; \lambda)=2 \int_{0}^{n}(\bar{n}-r)\left(\delta(r)-Y_{2}(r ; \lambda)\right) d r
$$




$$
\begin{aligned}
& =\int_{-\infty}^{\infty} \frac{\sin ^{2} \pi \bar{n} t}{\pi^{2} t^{2}}(1-b(t ; \lambda)) d t \\
& =\frac{1}{\pi^{2}} \int_{0}^{\infty}\left(\frac{1-\operatorname{con} \bar{n} t}{t}\right)\left(\frac{1-b(t / 2 \pi ; \lambda)}{(t / 2 \pi)}\right) d t \\
& =\Sigma^{2}(\bar{n} ; \infty)+\left[\Sigma^{2}(\bar{n} ; \lambda)-\Sigma^{2}(\bar{n} ; \infty)\right] .
\end{aligned}
$$

Here in the formal decomposition in the last form, the $\lambda$-independent term, namely the GUE result [22], is

$$
\begin{aligned}
\Sigma^{2}(\bar{n} ; \infty) & =\frac{1}{\pi^{2}} \int_{0}^{\infty}\left(\frac{1-\cos \bar{n} t}{t}\right)\left(\frac{1-b(t / 2 \pi ; \infty)}{t / 2 \pi)}\right) d t \\
& =\frac{1}{\pi^{2}} \int_{0}^{2 \pi}\left(\frac{1-\cos \bar{n} t}{t}\right) d t+\frac{1}{\pi^{2}} \int_{2 \pi}^{\infty}\left(\frac{2 \pi}{t}\right)\left(\frac{1-\cos \bar{n} t}{t}\right) d t \\
& =\frac{1}{\pi^{2}}\left[\log (2 \pi \bar{n})+\gamma+1-\operatorname{Ci}(2 \pi \bar{n})+2 \pi \bar{n}\left(\frac{\pi}{2}-\operatorname{Si}(2 \pi \bar{n})\right)-\cos 2 \pi \bar{n}\right] \\
& \rightarrow \frac{1}{\pi^{2}}[\log (2 \pi \bar{n})+\gamma+1]
\end{aligned}
$$

whereas the $\lambda$-dependent term is

$$
\begin{aligned}
\Sigma^{2}(\bar{n} ; \lambda)-\Sigma^{2}(\bar{n} ; \infty) & =\frac{1}{\pi^{2}} \int_{0}^{\infty}\left(\frac{1-\cos \bar{n} t}{t}\right)\left(\frac{b(t / 2 \pi ; \infty)-b(t / 2 \pi ; \lambda)}{t / 2 \pi}\right) d t \\
& =\frac{1}{\pi^{2}} \int_{0}^{\infty}\left(\frac{1-\cos \bar{n} t}{t}\right) e^{-4 \pi \lambda^{2} t}(1+h(t / 2 \pi ; \lambda)) d t \\
& =\frac{1}{2 \pi^{2}} \log \left(\frac{\bar{n}^{2}+16 \pi^{2} \lambda^{4}}{16 \pi^{2} \lambda^{4}}\right)+\xi(\lambda)+\rho(\bar{n} ; \lambda) \\
& \rightarrow \frac{1}{2 \pi^{2}} \log \left(\frac{\bar{n}^{2}+16 \pi^{2} \lambda^{4}}{16 \pi^{2} \lambda^{4}}\right)+\xi(\lambda) .
\end{aligned}
$$

The final asymptotic forms in $(6.11,6.12)$ are valid, with good approximations as in (6.2), for $\bar{n} \gtrsim 1$ and $\lambda$ of any size compared to $\bar{n}$. The functions $\xi(\lambda)$ and $\rho(\bar{n} ; \lambda)$ are defined by

$$
\xi(\lambda)=\frac{1}{\pi^{2}} \int_{0}^{\infty} e^{-4 \pi \lambda^{2} t} h(t / 2 \pi ; \lambda) \frac{d t}{t}
$$

and

$$
\begin{aligned}
\rho(\bar{n} ; \lambda) & =-\frac{1}{\pi^{2}} \int_{0}^{\infty} e^{-4 \pi \lambda^{2} t} \cos (\bar{n} t) h(t / 2 \pi ; \lambda) \frac{d t}{t} \\
& =-\frac{4 \lambda^{2}}{\bar{n}^{2}+16 \pi^{2} \lambda^{4}}+\ldots
\end{aligned}
$$

For small $\lambda$ we can use an expansion [23] of the $E$-function of (6.9). After some tedious calculations we get 


$$
\xi(\lambda)=\frac{1}{\pi^{2}} \log 4 \pi \lambda^{2}+\frac{1}{\pi^{2}}\left(\log 2 \pi+\gamma+1-\frac{\pi^{2}}{4}\right)-4 \lambda^{2}+\ldots
$$

which in $(6.10)$, along with $(6.11,6.12)$, reproduces for $\lambda=0$ the well-known GOE result [21]. We mention moreover that the final result in (6.9) has also been derived in [8] but without an exact form for $\xi(\lambda)$.

Another commonly-used fluctuation measure is the $\Delta_{3}$-statistic which for a given $\bar{n}$ measures the least square deviation of the staircase energy function from the best straight line fitting it $[21,22]$. Its ensemble average, related to $\Sigma^{2}[24]$, is given by

$$
\begin{aligned}
\bar{\Delta}_{3}(\bar{n} ; \lambda)= & \frac{2}{\bar{n}^{4}} \int_{0}^{\bar{n}}\left(\bar{n}^{3}-2 \bar{n}^{2} r+r^{3}\right) \Sigma^{2}(r ; \lambda) d r \\
\rightarrow & \frac{1}{2} \Sigma^{2}(\bar{n} ; \lambda)-\frac{9}{4 \pi^{2}}+\frac{4 \lambda^{4}}{\bar{n}^{2}}\left[1+\frac{2 \bar{n}}{\pi \lambda^{2}} \tan ^{-1}\left(\frac{\bar{n}}{4 \pi \lambda^{2}}\right)\right. \\
& \left.-4\left(1+\frac{4 \pi^{2} \lambda^{2}}{\bar{n}^{2}}\right) \log \left(\frac{\bar{n}^{2}+16 \pi^{2} \lambda^{4}}{16 \pi^{2} \lambda^{4}}\right)\right]
\end{aligned}
$$

where the last term in the last form goes from zero to $9 / 8 \pi^{2}$ as $\lambda$ increases from zero to infinity, agreeing with the known limiting results $[21,22]$. As indicated by the numerical integrations [5] for GOE and GUE, the term ignored, namely the integral involving $\rho(\bar{n} ; \lambda)$, may not however be very small for $\bar{n} \lesssim 10$.

\section{Appendix I. An Alternative Derivation of the Correlation and Cluster Functions}

An alternative way to derive the $n$-level functions is to consider the integral

$$
L_{N}(u)=\int_{-\infty}^{\infty} \cdots \int p\left(x_{1}, x_{2}, \ldots, x_{N}\right) \prod_{i=1}^{N} u\left(x_{i}\right) d x_{i}
$$

for an arbitrary function $u(x)$. This integral can be evaluated by expanding the pfaffian in (3.11) in terms of its elements and then integrating term by term by the method of integration over alternate variables as in [13]. We omit the details. The result for $N=2 m$ is

$$
L_{N}(u)=[\operatorname{det} \eta]^{1 / 2},
$$

where $\eta$ is an $N \times N$ antisymmetric matrix with elements $(i, j=0,1, \ldots, m-1)$

$$
\begin{gathered}
\eta_{2 i, 2 j}=2\left(\frac{1+\alpha^{2}}{1-\alpha^{2}}\right)^{i+j} \int_{-\infty}^{\infty} \int d x d y \varphi_{2 i}(x) \varphi_{2 j}(y) g(x-y) u(x) u(y), \\
\eta_{2 i, 2 j+1}=-\eta_{2 j+1,2 i}=\left(\frac{1+\alpha^{2}}{1-\alpha^{2}}\right)^{i-j} \int_{-\infty}^{\infty} \int d x d y \varphi_{2 i}(x) \psi_{2 j}(y) g(x-y) u(x) u(y), \\
\eta_{2 i+1,2 j+1}=\frac{1}{2}\left(\frac{1+\alpha^{2}}{1-\alpha^{2}}\right)^{-i-j} \int_{-\infty}^{\infty} \int d x d y \psi_{2 i}(x) \psi_{2 j}(y) g(x-y) u(x) u(y)
\end{gathered}
$$


The correlation functions now follow as functional derivatives of $L_{N}(u)$ with respect to $u$ at $u=1$ [13]. In particular the one and two level functions, Eqs. $(4.52,4.54)$, were derived in this way which facilitated the task of guessing the $N \times N$ quaternion matrix $\sigma^{(N)}$ in (4.12).

\section{Appendix II. Proof of Equations (4.30, 4.31)}

Consider the integral

$$
\begin{aligned}
& \int_{-\infty}^{\infty} \exp \left\{\left(2 y z-z^{2}\right)-\frac{1}{2}\left(1+\alpha^{2}\right) y^{2}-\left(\frac{1-\alpha^{4}}{4 \alpha^{2}}\right)(x-y)^{2}\right\} d y \\
& =\frac{2 \alpha \sqrt{\pi}}{1+\alpha^{2}} \exp \left\{-\left(\frac{1-\alpha^{2}}{1+\alpha^{2}}\right)^{2} z^{2}+2\left(\frac{1-\alpha^{2}}{1+\alpha^{2}}\right) x z-\frac{1}{2}\left(1-\alpha^{2}\right) x^{2}\right\} .
\end{aligned}
$$

Expanding both sides of this equation in powers of $z$, for which we use the generating function for the Hermite polynomials [25]

$$
\sum_{j=0}^{\infty} \frac{H_{j}(x)}{j !} z^{j}=e^{2 x z-z^{2}}
$$

and using the definition (4.18), we get

$$
\begin{gathered}
\int_{-\infty}^{\infty} \varphi_{j}(y) \exp \left\{-\frac{1}{2} \alpha^{2} y^{2}-\left(\frac{1-\alpha^{4}}{4 \alpha^{2}}\right)(x-y)^{2}\right\} \mathrm{dy} \\
=\left(\frac{2 \alpha \sqrt{\pi}}{1+\alpha^{2}}\right)\left(\frac{1-\alpha^{2}}{1+\alpha^{2}}\right)^{j} e^{(1 / 2) \alpha^{2} x^{2}} \varphi_{j}(x) .
\end{gathered}
$$

Then a partial integration along with the definitions $(4.17,4.19)$ yields $(4.30)$.

For (4.31), it is convenient to write it as

$$
\frac{1}{2}\left(\frac{1+\alpha^{2}}{1-\alpha^{2}}\right)^{j+(1 / 2)} \int_{-\infty}^{\infty} f(x-y) e^{-(1 / 2) \alpha^{2} y^{2}} \varphi_{j}(y) d y=\int_{-\infty}^{\infty} \in(x-y) e^{(1 / 2) \alpha^{2} y^{2}} \varphi_{j}(y) d y,
$$

where we have used the definition (4.20). Differentiating both sides of this relation with respect to $x$, we obtain (II.3). Therefore the relation is true if it is valid for one value of $x$. At $x=\infty, f(x-y)=2 \in(x-y)=1$, so that we have to prove that

$$
\left(\frac{1+\alpha^{2}}{1-\alpha^{2}}\right)^{j+(1 / 2)} \int_{-\infty}^{\infty} e^{-(1 / 2) \alpha^{2} y^{2}} \varphi_{j}(y) d y=\int_{-\infty}^{\infty} e^{(1 / 2) \alpha^{2} y^{2}} \varphi_{j}(y) d y .
$$

When $j$ is odd, the truth of this equation is evident. For even $j$, we consider the integral

$$
\int_{-\infty}^{\infty} \exp \left\{\left(2 y z-z^{2}\right)-\frac{1}{2}\left(1 \pm \alpha^{2}\right) y^{2}\right\} d y=\left(\frac{2 \pi}{1 \pm \alpha^{2}}\right)^{1 / 2} \exp \left\{\left(\frac{1-\alpha^{2}}{1+\alpha^{2}}\right)^{ \pm 1} z^{2}\right\}
$$


which along with (II.2) yields

$$
\int_{-\infty}^{\infty} e^{ \pm(1 / 2) \alpha^{2} y^{2}} \varphi_{2 j}(y) d y=\left(\frac{2 \pi}{1 \pm \alpha^{2}}\right)^{1 / 2}\left(2^{2 j}(2 j) ! \sqrt{\pi}\right)^{-(1 / 2)} \frac{(2 j) !}{j !}\left(\frac{1-\alpha^{2}}{1+\alpha^{2}}\right)^{ \pm j}
$$

verifying (II.5). This completes the proof of (4.31).

\section{Appendix III}

Asymptotic forms of $J(r ; \lambda)$ and $D(r ; \lambda)$.

By partial integration

$$
\begin{aligned}
\int_{0}^{\pi} \frac{\sin k r}{k} \exp \left(-2 \lambda^{2} k^{2}\right) d k= & \exp \left(-r^{2} / 8 \lambda^{2}\right) \operatorname{Im} \int_{\pi}^{\infty} \exp -\left\{-2 \lambda^{2}\left(k-\frac{i r}{4 \lambda^{2}}\right)^{2}\right\} \frac{d k}{k} \\
= & \exp \left(-r^{2} / 8 \lambda^{2}\right) \operatorname{Im}\left[\exp \left\{-2 \lambda^{2}\left(k-\frac{i r}{4 \lambda^{2}}\right)^{2}\right\}\right. \\
& \left.\left.\times\left\{-\frac{1}{4 \lambda^{2} k\left(k-\frac{i r}{4 \lambda^{2}}\right)}+\ldots\right\}\right]_{\pi}^{\infty}\right) \\
= & \exp \left(-2 \lambda^{2} \pi^{2}\right) \operatorname{Im} e^{i \pi r}\left\{\frac{-1}{\pi\left(4 \pi \lambda^{2}-i r\right)}+\ldots\right\} \\
= & \frac{1}{\pi} \exp \left(-2 \lambda^{2} \pi^{2}\right)\left\{\frac{r \cos \pi r+4 \pi \lambda^{2} \sin \pi r}{16 \pi^{2} \lambda^{4}+r^{2}}+\ldots\right\} .
\end{aligned}
$$

Similarly

$$
\begin{gathered}
\int_{0}^{\infty} k \sin k r \exp \left(2 \lambda^{2} k^{2}\right) d k \\
=\pi \exp \left(2 \lambda^{2} \pi^{2}\right)\left\{\frac{r \cos \pi r-4 \pi \lambda^{2} \sin \pi r}{16 \pi^{2} \lambda^{4}+r^{2}}+\ldots\right\} .
\end{gathered}
$$

Eqs. $(5.16,5.18,6.1)$ then give (6.2).

Acknowledgements. We are grateful to O. Bohigas for a critical reading of the manuscript, and to J. B. French and V. K. B. Kota for unpublished results. We are also indebted to D. Bessis, J. des Cloizeaux and B. Derrida for various helpful discussions.

\section{References}

1. Porter, C. E. (ed.): Statistical theories of spectra: Fluctuations. New York: Academic Press 1965

2. Wigner, E. P.: SIAM Rev. 9, 1 (1967)

3. Mehta, M. L.: Random matrices and the statistical theory of energy levels. New York: Academic Press 1967

4. Brody, T. A., Flores, J., French, J. B., Mello, P. A., Pandey, A., Wong, S. S. M.: Random-matrix physics: Spectrum and strength fluctuations. Rev. Mod. Phys. 53, 385 (1981); this review covers recent developments and contains up to date references in the field. 
5. Haq, R. U., Pandey, A., Bohigas, O.: Fluctuation properties of nuclear energy levels: Do theory and experiment agree? Phys. Rev. Lett., 48, 1086 (1982)

6. Dyson, F. J.: Statistical theory of the energy levels of complex systems. I. J. Math. Phys. 3, 140 (1962), reprinted in Porter [1]; Mehta, M. L., Ref. [3], Chap. 2

7. Favro, L. D., McDonald, J. F.: Possibility of detecting a small time-reversal-noninvariant term in the Hamiltonian of a complex system by measurements of energy-level spacings. Phys. Rev. Lett. 19, 1254 (1967); Rosenzweig, N., Monahan, J. E., Mehta, M. L.: Perturbation of the statistical properties of nuclear states and transitions by interactions that are odd under time reversal. Nucl. Phys. A109, 437 (1968); McDonald, J. F.: On the width distribution for a complex system whose Hamiltonian contains a small interaction that is odd under time reversal. J. Math. Phys. 10, 1191 (1969); Mehta, M. L.: Matrices hermitiques aléatoires à partie imaginaire petite. Nuovo Cim B65, 107 (1970); Camarda, H. S.: Upper limit on a time reversal noninvariant part of Wigner's random matrix model. Phys. Rev. C13, 2524 (1976); McDonald, J. F.: Some mathematically simple ensembles of random matrices which represent Hamiltonians with a small time reversal noninvariant part. Nuovo Cimento B57, 95 (1980)

8. French, J. B., Kota, V.K.B., Pandey, A.: Upper bound for time reversal non-invariance from energy level fluctuations. (to be published)

9. Pandey, A.: Statistical Properties of many particle spectra IV. New ensembles by Stieltjes transform methods. Ann. Phys. (N. Y.) 134, 110 (1981)

10. See for example Mehta, M. L.: Ref. [3], Chap. 3

11. Mehta, M. L., Rosenzweig, N.: Distribution laws for the roots of a random antisymmetric hermitian matrix. Nucl. Phys. A109, 449 (1968)

12. Itzykson, C., Zuber, J. B.: The planar approximation. II.J. Math. Phys. 21, 411 (1980); Mehta, M. L.: A method of integration over matrix variables. Commun. Math. Phys. 79, 327 (1981)

13. See for example Mehta, M. L.: Ref. [3], Sect. 5.2, appendix A.7

14. Dyson, F. J.: Correlations between eigenvalues of a random matrix. Commun. Math. Phys. 19, 235 (1970); Mehta, M. L.: Elements of matrix theory, Delhi: Hindustan Publishing Corporation, 1977, Chap. 8

15. Mehta, M. L.: A note on correlations between eigenvalues of a random matrix. Commun. Math. Phys. 20, 245 (1971)

16. Dyson, F. J.: Statistical theory of the energy levels of complex systems. III. J. Math. Phys. 3, 166 (1962), reprinted in Porter [1]; Mehta, M. L.: Ref. [3], Chap. 5

17. Pandey, A.: Statistical properties of many-particle spectra III. Ergodic behavior in random-matrix ensembles. Ann. Phys. (N. Y.) 119, 170 (1979), Appendix I

18. Szegö, G.: Orthogonal Polynomials, New York: Am. Math. Soc., 1939, Sect. 8.22, page 195

19. Bateman, H.: Tables of integral transforms. Erdelyi, A., (ed.), Vol. 1 pp. 18 and 96. New York: McGraw Hill, 1954

20. Mehta, M. L.: Ref. [3], Appendix A. 10

21. Dyson, F. J., Mehta, M. L.: Statistical theory of energy levels of complex systems. IV, J. Math. 4, 701 (1963), reprinted in Porter [1]

22. Brody, T. A., et al.: Ref. [4], Sects, V. C., V. D.

23. Abramowitz, M., Stegun, I. A., (eds.): Handbook of Mathematical Functions, Washington: National Bureau of Standards, U. S. Department of Commerce, 1964, Chap. 5, Eqs. (5.1.11, 5.1.32, 5.2.1, 5.2.2)

24. Pandey, A.: Ref. [17], Appendix II

25. Abramowitz, M., Stegun, I. A.: Ref. [23], Eq. (22.9.17)

Communicated by $\mathrm{H}$. Araki

Received May 4, 1982

Note added in proof. The ensemble of Hermitian matrices $H$ with $\|\operatorname{Im} H\|^{2}>\|\operatorname{Re} H\|^{2}$, corresponding to $\alpha^{2}>1$, has now been studied. We find the same joint probability density for the eigenvalues as in (3.11), and hence all the results which follow from it. 